\title{
fib-tf: A TensorFlow-based Cardiac Electrophysiology Simulator
}

\section{Shahriar Iravanian ${ }^{1}$}

1 Emory University

\section{Summary}

- Repository ¿

- Archive ct

Submitted: 25 April 2018

Published: 01 June 2018

\section{Licence}

Authors of papers retain copyright and release the work under a Creative Commons Attribution $4.0 \mathrm{In}$ ternational License (CC-BY). $\mathrm{fib}$ tf is a Python package developed on top of the machine-learning library TensorFlow for cardiac electrophysiology simulation (Abadi et al. 2015). While TensorFlow is primarily designed for machine learning, it also provides a framework to perform general-purpose multidimensional tensor manipulation.

The primary goal of $f i b \_t f$ is to test and assess the suitability of TensorFlow for solving systems of stiff ordinary differential equations (ODE), such as those encountered in cardiac modeling. It mainly targets massively parallel hardware architectures (e.g., Graphics Processing Units).

fib_tf solves the monodomain reaction-diffusion equations governing cardiac electrical activity by a combination of the finite-difference and explicit Euler methods. It is used to simulate two cardiac ionic models: the 4-variable Cherry-Ehrlich-Nattel-Fenton canine left-atrial and the 8-variable Beeler-Reuter ventricular models (Cherry et al. 2007; Beeler and Reuter 1977).

fib_tf serves as a testbed to try various general and TensorFlow-specific optimization techniques. We showed that enabling Just-In-Time (JIT) compilation significantly improves the performance. Moreover, by applying a multitude of optimization methods, including dataflow graph unrolling, the Rush-Larsen method (Rush and Larsen 1978), the Chebyshev polynomials approximation, and multi-rate integration, we have achieved a performance within a factor 2-3 of hand-optimized CUDA codes. The motivation behind and the details of each method are described in the documentation.

Based on our experiments, TensorFlow applicability is not limited to the machine-learning domain. TensorFlow is a valuable tool for the development of efficient and complex ODE solvers. fib_tf can act as a framework and model for such solvers. Especially, it is useful in rapid prototyping and testing of new algorithms and methods.

\section{Acknowledgements}

\section{References}

Abadi, Martín, Ashish Agarwal, Paul Barham, Eugene Brevdo, Zhifeng Chen, Craig Citro, Greg S. Corrado, et al. 2015. "TensorFlow: Large-Scale Machine Learning on Heterogeneous Systems." https://www.tensorflow.org/.

Beeler, G W, and H Reuter. 1977. "Reconstruction of the action potential of ventricular myocardial fibres." The Journal of Physiology 268 (1):177-210. https://doi.org/10.1113/ jphysiol.1977.sp011853. 
Cherry, Elizabeth M., Joachim R. Ehrlich, Stanley Nattel, and Flavio H. Fenton. 2007. "Pulmonary vein reentry-Properties and size matter: Insights from a computational analysis." Heart Rhythm 4 (12):1553-62. https://doi.org/10.1016/j.hrthm.2007.08.017.

Rush, Stanley, and Hugh Larsen. 1978. "A Practical Algorithm for Solving Dynamic Membrane Equations." IEEE Transactions on Biomedical Engineering BME-25 (4):38992. https://doi.org/10.1109/TBME.1978.326270. 